This article has been accepted for publication in Monthly Notices of the Royal Astronomical Society (C): 2018 The Authors. Published by Oxford University Press on behalf of the Royal Astronomical Society. All rights reserved. 


\title{
Flux-ratio anomalies from discs and other baryonic structures in the Illustris simulation
}

\author{
Jen-Wei Hsueh, ${ }^{1 \star}$ Giulia Despali, ${ }^{2}$ Simona Vegetti, ${ }^{2}$ Dandan $\mathrm{Xu},{ }^{3}$ \\ Christopher D. Fassnacht ${ }^{1}$ and R. Benton Metcalf ${ }^{4,5}$ \\ ${ }^{1}$ Physics Department, University of California, Davis, 1 Shields Ave., Davis, CA 95616, USA \\ ${ }^{2}$ Max Planck Institute for Astrophysics, Karl-Schwarzschild-Strasse 1, D-85740 Garching, Germany \\ ${ }^{3}$ Heidelberg Institute for Theoretical Studies, Schloss-Wolfsbrunnenweg 35, D-69118 Heidelberg, Germany \\ ${ }^{4}$ Dipartimento di Fisica e Astronomia, Universita di Bologna, Via Gobetti 93/2, I-40129 Bologna, Italy \\ ${ }^{5}$ INAF - Osservatorio Astronomico di Bologna, Via Gobetti 93/3, I-40129 Bologna, Italy
}

Accepted 2017 December 21. Received 2017 December 5; in original form 2017 July 23

\begin{abstract}
The flux ratios in the multiple images of gravitationally lensed quasars can provide evidence for dark matter substructure in the halo of the lensing galaxy if the flux ratios differ from those predicted by a smooth model of the lensing galaxy mass distribution. However, it is also possible that baryonic structures in the lensing galaxy, such as edge-on discs, can produce fluxratio anomalies. In this work, we present the first statistical analysis of flux-ratio anomalies due to baryons from a numerical simulation perspective. We select galaxies with various morphological types in the Illustris simulation and ray trace through the simulated haloes, which include baryons in the main lensing galaxies but exclude any substructures, in order to explore the pure baryonic effects. Our ray-tracing results show that the baryonic components can be a major contribution to the flux-ratio anomalies in lensed quasars and that edge-on disc lenses induce the strongest anomalies. We find that the baryonic components increase the probability of finding high flux-ratio anomalies in the early-type lenses by about 8 percent and by about $10-20$ percent in the disc lenses. The baryonic effects also induce astrometric anomalies in 13 per cent of the mock lenses. Our results indicate that the morphology of the lens galaxy becomes important in the analysis of flux-ratio anomalies when considering the effect of baryons, and that the presence of baryons may also partially explain the discrepancy between the observed (high) anomaly frequency and what is expected due to the presence of subhaloes as predicted by the cold dark matter simulations.
\end{abstract}

Key words: gravitational lensing: strong-galaxies: structure.

\section{INTRODUCTION}

One of the key probes for investigating the nature of dark matter is a well-constrained determination of the mass function of substructure associated with galaxy-scale haloes. Strong gravitational lensing is an especially powerful tool for detecting substructure in distant galaxies, which is achieved via two main approaches, namely the gravitational imaging technique and the analysis of fluxratio anomalies. The gravitational imaging technique (Koopmans 2005; Vegetti \& Koopmans 2009) focuses on systems in which the background galaxy is lensed into a long arc or Einstein ring. Substructures that are located close to the arcs or ring produce small astrometric perturbations to the lensed emission, which can be detected as a residual when comparing the observed surface brightness

^E-mail: jwhsueh@ucdavis.edu distribution to that predicted by a smooth (i.e. without substructure) mass lens model. Several substructures with $10^{8}-10^{9} \mathrm{M}_{\odot}$ have now been detected with this technique (Vegetti et al. 2010, 2012; Hezaveh et al. 2016). With the gravitational imaging technique, the projected position and mass of the substructure can be determined. The minimum detectable substructure mass is determined by the source size, surface brightness structure of the source, and the angular resolution of the imaging. Thus, this technique requires highresolution imaging. The inferred substructure abundances from current samples that have been analysed with the gravitational imaging technique are marginally consistent with those predicted by dark matter only and hydrodynamical simulations although the sample size is limited (Vegetti et al. 2014; Despali \& Vegetti 2017).

The other approach, the analysis of flux-ratio anomalies, was first proposed by Mao \& Schneider (1998) and Metcalf \& Madau (2001). In this case, the targeted systems show multiple lensed images of a background active galactic nucleus (AGN). Deviations 
between the ratios of the observed fluxes of the lensed AGN and those predicted by smooth lens models - called 'flux-ratio anomalies' - can be caused by small-scale structure in the halo of the lensing galaxy such as dark matter substructure. In particular, in four-image lens systems the flux ratios of 'merging images' (i.e. those that are very close together on the sky) follow relations that are nearly universal for smooth mass models. These flux ratios are sensitive to perturbations in the lensing potential. To reliably quantify the perturbation effect from substructures, it is necessary to obtain flux measurements that are as free as possible from nongravitational effects. In practice, this often means fluxes that have been measured at radio wavelengths and with long-term monitoring (in order to average out the intrinsic variation of the lensed AGN). This is because the radio emission region in quasars is much larger than the Einstein radius from stars in the foreground lensing galaxy, and thus the radio fluxes are free from the stellar microlensing that often seen at optical wavelengths (but also see Koopmans \& de Bruyn 2000, for a rare case.) Also, observing at radio wavelengths can minimize dust extinction, which affects flux ratios at shorter wavelengths. Although propagation effects such as interstellar scattering and free-free absorption can influence the radio wavelength flux ratios, their frequency dependence are well understood (Winn, Rusin \& Kochanek 2004; Mittal, Porcas \& Wucknitz 2007).

Dalal \& Kochanek (2002) presented the first statistical results from the flux-ratio technique, using a sample of seven radio-loud lensed quasars. Their inferred substructure abundance is consistent within the errors with the predictions from cold dark matter (CDM) cosmology, although the monitoring in the flux measurements was not done yet at that time. In the follow-up work of Kochanek \& Dalal (2004), they explore alternative sources of fluxratio anomalies and conclude that these alternatives are less likely to be the cause of the observed anomalies. The assumption that the flux-ratio anomalies in radio-loud lenses are generated solely by the substructure then became the standard in subsequent studies, such as for example, Bradač et al. (2002), Metcalf \& Zhao (2002), Dobler \& Keeton (2006), Fadely \& Keeton (2012) and Nierenberg et al. (2014) (however see Nierenberg et al. 2017, for the case of no detection.) However, it is not entirely clear whether the CDM substructure scenario is fully compatible with current flux anomaly observations. Numerical studies based on CDM simulations (e.g. Mao et al. 2004; Macciò \& Miranda 2006; Xu et al. 2009, 2015; Chen, Koushiappas \& Zentner 2011; Metcalf \& Amara 2012) show that the predicted CDM substructure population is not sufficient to reproduce the high flux-ratio anomaly strengths currently observed. This result suggests that assuming substructures are the only source of flux-ratio anomalies may be oversimplified.

Complex baryonic structures in the lens galaxies may provide a viable explanation for the observed high anomaly strength. For example, Möller, Hewett \& Blain (2003) and Quadri, Möller \& Natarajan (2003) have discussed the influence of disc structure on flux ratios and Hsueh et al. $(2016,2017)$ have recently shown that with the inclusion of an edge-on disc in the lens model, motivated by high-resolution imaging, the observed flux ratios and positions of lensed images can be successfully reproduced without the need for substructure. Gilman et al. (2017) have also shown that in elliptical lenses, 10-15 percent of anomalies are generated from the baryonic structures in the lens galaxies (see also Xu et al. 2010, from the simulation perspective). Moreover, line-of-sight structures can also contribute to the flux-ratio anomalies (Metcalf 2005; Xu et al. 2012; McCully et al. 2014). These alternative sources of fluxratio anomalies may explain why the CDM substructure abundance predicted by $N$-body simulations at galactic- and group-scales can- not reproduce the observed high frequency of flux-ratio anomalies (Xu et al. 2009, 2015).

In this paper, we explore the effect of stellar discs and other baryonic structures on flux-ratio anomalies using a state-of-the-art cosmological hydrodynamical simulation - the Illustris simulation (Genel et al. 2014; Vogelsberger et al. 2014a,b; Nelson et al. 2015). This paper is organized as follows. In Section 2, we describe the simulation, the criteria for selecting lenses we use for this work, and the final sample of galaxies selected from the simulation. In Section 3, we present the ray-tracing results for smooth mass models and the simulated lenses. In Section 4, we discuss the flux-ratio and astrometric anomalies in our mock lenses. Finally, in Section 5, we summarize the results in this work.

\section{SIMULATED LENSES}

The goal of this paper is to investigate the effect of edge-on discs and other baryonic structures on the flux ratios of gravitationally lensed quasars. Recent hydrodynamical simulations are able to produce a realistic population of galaxies, with a variety of morphological types, and so constitute the ideal tool for our purposes. The first step of our work is then to construct a sample of simulated lens galaxies from the Illustris simulation. The Illustris Project is a series of hydrodynamical simulations of cosmological volumes that follow the evolution of dark matter, cosmic gas, stars, and supermassive black holes from a starting redshift of $z=127$ to the present time. At the highest resolution level, which we used in this work, the simulation covers a cosmological volume of $(106.5 \mathrm{Mpc})^{3}$ and has a mass resolution of $6.26 \times 10^{6}$ and $1.26 \times 10^{6} \mathrm{M}_{\odot}$ for dark matter and baryons, respectively. The simulations were run using the recent moving-mesh AREPO code (Springel 2010) and the adopted cosmological model has $\Omega_{\mathrm{m}}=0.2726, \Omega_{\Lambda}=0.7274, \Omega_{\mathrm{b}}=0.0456$, $h=0.704$, and $\sigma_{8}=0.809$, consistent with the 9-year Wilkinson Microwave Anisotropy Probe (WMAP-9) measurements (Hinshaw et al. 2013). More details on the simulation and the implementation of baryonic physics can be found in Vogelsberger et al. (2014a,b), Genel et al. (2014), and Nelson et al. (2015).

In order to select a sample of disc galaxies compatible with the available observational data (e.g. Patnaik et al. 1992; Browne et al. 1998, 2003; Wilkinson et al. 1998; Myers et al. 2003; Chiba et al. 2005; Minezaki et al. 2009), we focus on objects at redshift $z_{1}=0.6$. At first, we apply the following general selection criteria: (i) a galaxy must be the central galaxy of the considered host halo; (ii) the dark matter halo must be less massive than $5 \times 10^{13} \mathrm{M}_{\odot}$ since above this halo mass, galaxies are in a cluster environment; (iii) a galaxy must have a stellar mass $M_{*}>10^{10} \mathrm{M}_{\odot}$ : this corresponds to $\sim 10000$ stellar particles that are sufficient to reliably estimate the broad galaxy morphology ('elliptical' versus 'disc') by resolving the radial surface brightness and calculating the internal kinematics. In addition, we apply two further selection criteria - one based on morphology and the other on stellar kinematic information - in order to identify a disc galaxy sample and its early-type counterpart. We describe these selection criteria in more detail in the following sections.

\subsection{Morphological criteria}

For the morphology-based selections, the galaxy classifications and Sérsic profile fitting results were taken from Xu et al. (2017), where the morphological type of each galaxy was determined by fitting both the de Vaucouleurs profile and the exponential profile to the radial surface brightness distribution of the elliptical isophotes. Fig. 1 

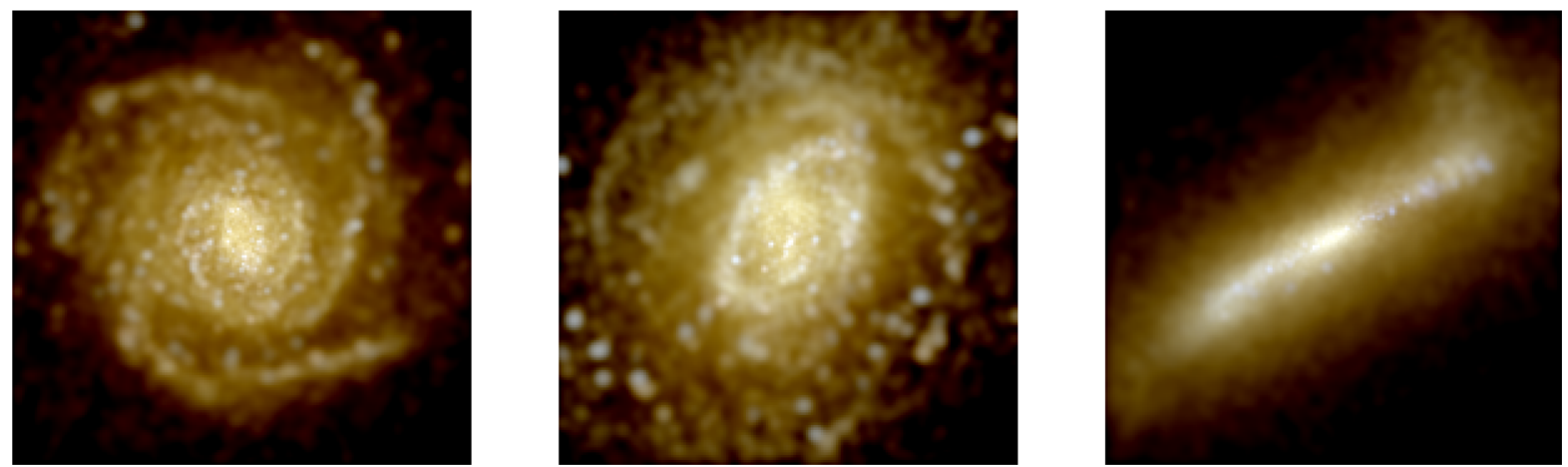

Figure 1. Synthesized images of Illustris disc galaxies with different inclination angles $(i)$. Left: $i=7^{\circ}$; centre: $i=50^{\circ}$; right: $i=80^{\circ}$. The images are produced by combining the surface brightness maps in the rest-frame SDSS $g, r$, and $i$ filter bandpasses (see Xu et al. 2017, for more details).

presents the synthesized images of some examples of disc galaxies in the Illustris simulation. The images are produced by combining the surface brightness maps in the rest-frame Sloan Digital Sky Survey (SDSS) $g, r$, and $i$ filter bandpasses (see Xu et al. 2017, for more details). A galaxy is classified as an early-type if the de Vaucouleurs profile provides a better fit, while if the exponential profile is a better fit then the galaxy is classified as a late-type. Thus, to further restrict our disc galaxy sample, we also require that the selected disc galaxy must also have a Sérsic index of $n<2$. Galaxy types defined in this way are referred to as 'morphology-selected' types.

\subsection{Kinematic criteria}

From a kinematic point of view, a stellar disc is a group of stars that corotates around the symmetric axis (we will call this symmetric axis the ' $z$-axis', for simplicity). By looking into the kinematic properties of each stellar particle, a simulated galaxy can be decomposed into a thin disc, a thick disc, and a bulge. This dynamical decomposition is usually done in the parameter space of the $z$-component of the specific angular momentum, $J_{z}$, and the specific binding energy, $E$, of each star (Abadi et al. 2003). On the $J_{z}-E$ plane, stellar and gaseous components corotate, which defines a solid curve $J_{\text {circ }}(E)$ (see fig. 2 in Abadi et al. 2003) that represents the corotating circular orbits in the disc as a function of specific binding energy. The circularity parameter $\epsilon_{z}$ of each star is defined as the ratio between its $J_{z}$ and corresponding $J_{\text {circ }}(E)$, showing how closely a stellar or gaseous component follows the circular motion of the disc. In general, the gaseous disc and the thin disc stellar components have a sharp distribution of $\epsilon_{z}$ peaked about unity. Based on the $\epsilon_{z}$ distribution, all stars within the 'luminous' radius can be decomposed into three different dynamical components: a bulge, a thin disc, and a thick disc. For the Illustris simulation, the quantity $\epsilon$ has been calculated for all particles and for each galaxy the following quantities are provided (see Genel et al. 2015, for more details):

(i) the fraction of stars with $\epsilon_{z}>0.7$, which can be used to identify the thin disc stars - those with significant (positive) rotational support;

(ii) the fraction of stars with $\epsilon_{z}<0$, multiplied by 2 , which in turn defines the mass fraction of the bulge.

Following Teklu et al. (2015), galaxies with more than 40 per cent of stars in the thin disc (above-mentioned fraction 1) are considered as 'kinematics-selected' disc galaxies. On the other hand, galaxies with more than 60 per cent of stars in the bulge (above-mentioned fraction 2) are considered as 'kinematics-selected' elliptical/earlytype galaxies.

\subsection{Disc and elliptical lens samples in this work}

Here we describe the final galaxy samples used in this work and we summarize their distributions. Fig. 2 shows the dependence on stellar mass (left-hand panel) and on Einstein radius ${ }^{1}$ (right-hand panel) of the fractions of galaxies that are defined as one of the following types: morphology-selected discs, kinematics-selected discs, and elliptical galaxies, at $z=0.6$ from the Illustris simulation. The source redshift is fixed at $z_{\mathrm{s}}=2.0$ to maximize the Einstein radius for the simulated lenses. Note that the histograms in Fig. 2 do not sum up to 1.0 because some lens galaxies satisfy both the morphological and kinematic criteria, and some other galaxies do not meet either criteria. However, it is still clear from the distributions that the majority of lens systems with small Einstein radii are produced by disc galaxies, as expected (e.g. Turner, Ostriker \& Gott 1984). We note that the selection based on kinematics is more stringent than the morphological one, as it requires the formation of well-established discs; as a consequence, the number of kinematics-selected galaxies is small, especially in the low-mass regime (also see Bottrell et al. 2017). In our final disc sample, we only include those galaxies that satisfy both criteria.

For each selected disc galaxy, we calculate an inclination angle, which is defined as the angle between the line of sight and the major rotational axis, i.e. the ' $z$-axis'. From the visualization of simulated galaxies, we further define a subsample of edge-on discs whose inclination angles are larger than $80^{\circ}$ and a subsample of face-on discs whose inclination angles are smaller than $50^{\circ}$. Fig. 1 presents the synthesized images of example disc galaxies of different inclination angles from the Illustris simulation.

Our final elliptical sample includes galaxies that simultaneously satisfy: (1) having a better fit to the de Vaucouleurs profile in surface brightness; (2) Sérsic index larger than 2; and (3) more than 60 per cent of stars in the bulge. A summary of the selection criteria for different galaxy types is given in Table 1.

The left-hand panel of Fig. 3 shows the distribution of stellar mass versus Einstein radius for disc lenses and elliptical lenses that satisfy the selection criteria as listed in Table 1, as well as all other lenses that do not fit to our definition of either disc or elliptical galaxies. The right-hand panel of Fig. 3 shows the distribution of the disc mass versus the Einstein radius for all disc lens candidates

\footnotetext{
${ }^{1}$ The Einstein radius $R_{\mathrm{E}}$ of each galaxy is calculated as the projected radius within which the mean surface mass density $\bar{\Sigma}\left(\leqslant R_{\mathrm{E}}\right)$ is equal to the critical density $\Sigma_{\mathrm{cr}}=\frac{C^{2}}{4 \pi G} \frac{D_{\mathrm{s}}}{D_{\mathrm{ds}} D_{\mathrm{d}}}$, where $D_{\mathrm{d}}, D_{\mathrm{s}}$, and $D_{\mathrm{ds}}$ are the angular diameter distances from the observer to the lens, to the source, and from the lens to the source, respectively.
} 

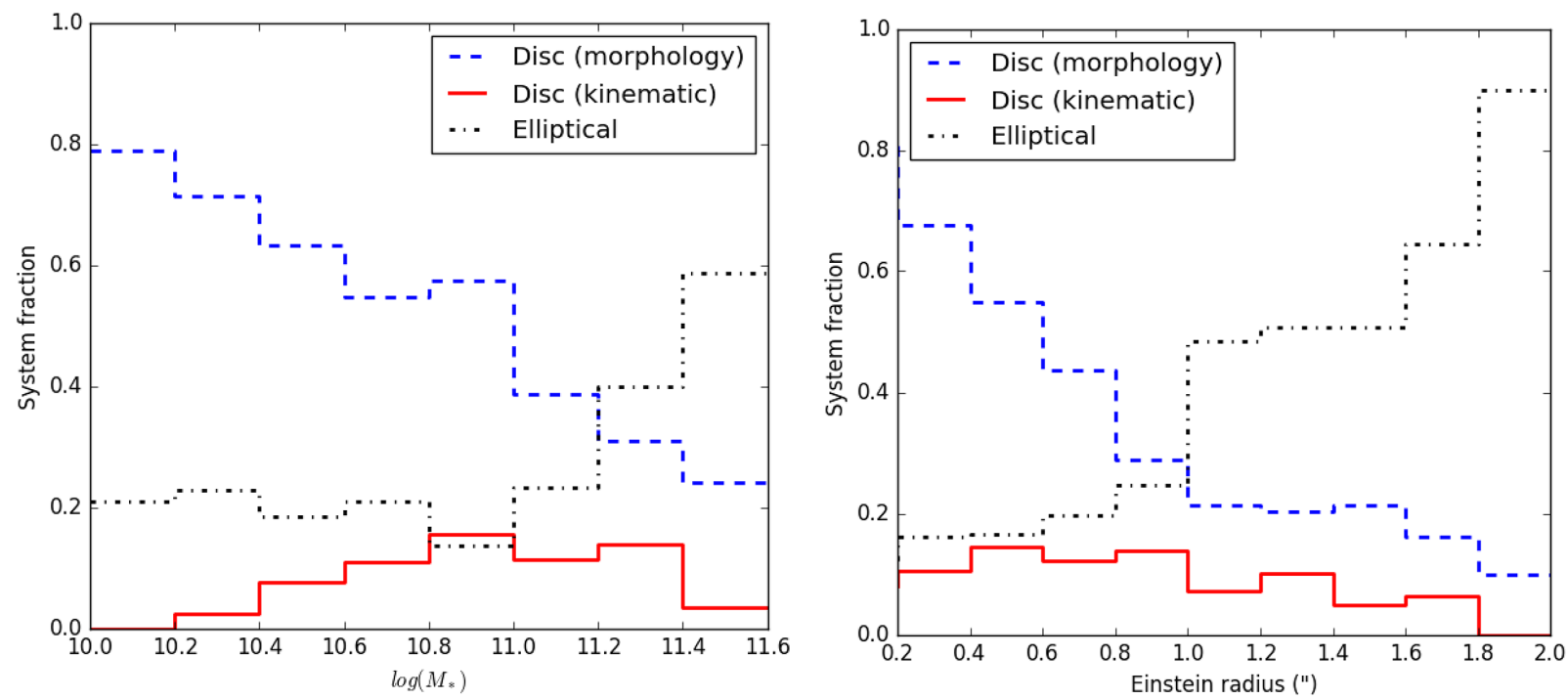

Figure 2. Left: lens system fraction versus stellar mass $\left(\mathrm{M}_{\odot} h^{-1}\right)$ in Illustris at redshift $z=0.6$. The morphology-selected disc lenses are shown in the blue dashed line, kinematics-selected disc lenses are shown in the red solid line, and elliptical lenses are shown in the black dot-dashed line. Right: lens system fraction versus Einstein radius (in arcsecond) with source redshift assigned as $z_{\mathrm{s}}=2.0$. Note that some lens galaxies satisfy both the morphological and kinematic criteria, and some galaxies do not meet either disc or elliptical criteria.

Table 1. Summary of the selection criteria. Galaxies of each type must satisfy all the criteria listed.

\begin{tabular}{lc}
\hline Disc galaxy & Elliptical galaxy \\
\hline Exponential disc profile & De Vaucouleur profile \\
Sèrsic index $<2$ & Sèrsic index $>2$ \\
Disc star fraction $>40$ per cent & Bulge star fraction $>60$ per cent \\
Face-on: $i<50^{\circ}$ & \\
Edge-on: $i>80^{\circ}$ & \\
\hline
\end{tabular}

in Illustris at $z=0.6$, where edge-on and face-on discs are labelled as red dots and green diamonds, respectively. At fixed stellar-disc mass, the edge-on disc lenses tend to have larger Einstein radii than the face-on disc lenses, which indicates that the edge-on disc galaxies are more efficient lenses. Our analysis focuses on lenses with Einstein radii larger than 0.3 arcsec, in order to match the current observational limitations. Because of computational limitations, for our ray-tracing experiment we randomly select subsets of galaxies of different types. Our complete ray-tracing samples comprise the following four groups: 50 elliptical galaxies, 25 edge-on disc galaxies, 25 face-on disc galaxies, and a sample of 50 disc lenses that
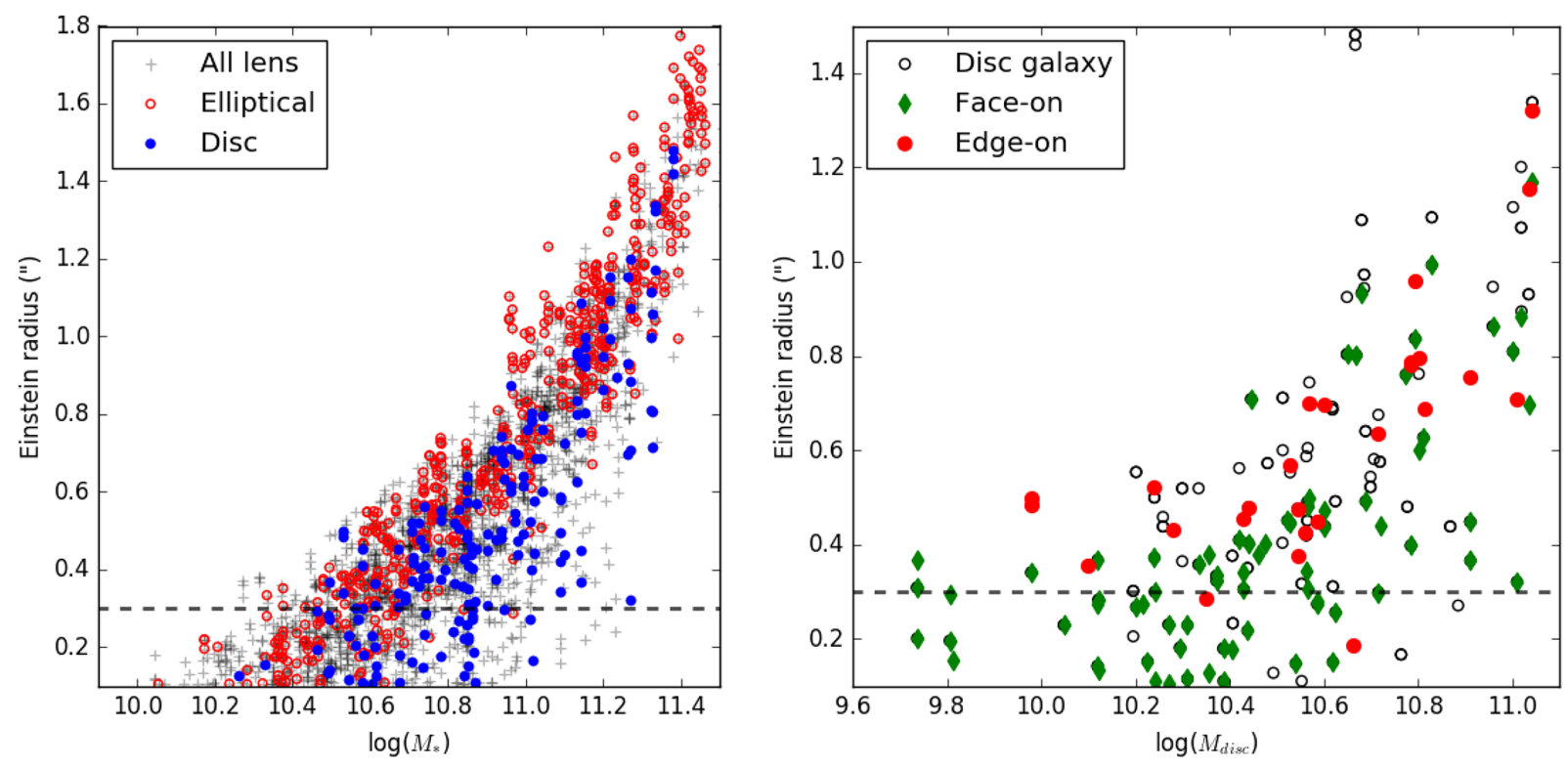

Figure 3. Left: scatter plot of stellar mass $\left(h^{-1} \mathrm{M}_{\odot}\right)$ and Einstein radius (arcsecond) for all lenses at redshift $z=0.6$ in Illustris, which are shown as black crosses. All elliptical lenses are shown as red open circles, while disc lenses are shown as blue circles. The Einstein radius cut, $\theta_{\mathrm{E}}>0.3$ arcsec, is shown with the grey dashed line. Right: scatter plot of disc mass $\left(h^{-1} \mathrm{M}_{\odot}\right.$ ) versus Einstein radius (arcsecond) of all disc lenses as open black circles, face-on disc lenses as green diamonds, and edge-on disc lenses as red circles. 
includes members of both the edge-on and face-on samples plus disc galaxies with $50<i<80$.

\section{FLUX-RATIO ANOMALIES IN ILLUSTRIS}

Lenses with small opening angles in the fold and cusp configurations are the most sensitive to the perturbations of the gravitational potential that are responsible for flux-ratio anomalies. The relations $R_{\text {fold }}$ and $R_{\text {cusp }}$, which can be used to describe the anomaly strengths, are given below (Blandford \& Narayan 1986; Mao 1992; Schneider \& Weiss 1992; Zakharov 1995; Koopmans et al. 2003):

$R_{\mathrm{fold}}=\frac{\mu_{\mathrm{A}}+\mu_{\mathrm{B}}}{\left|\mu_{\mathrm{A}}\right|+\left|\mu_{\mathrm{B}}\right|}$

and

$R_{\text {cusp }}=\frac{\mu_{\mathrm{B}}+\mu_{\mathrm{A}}+\mu_{\mathrm{C}}}{\left|\mu_{\mathrm{A}}\right|+\left|\mu_{\mathrm{B}}\right|+\left|\mu_{\mathrm{C}}\right|}$,

where $\mu_{\mathrm{A}, \mathrm{B}}$ in $R_{\text {fold }}$ are the magnifications of the two lensed images that make up the merging double, and $\mu_{\mathrm{A}, \mathrm{B}, \mathrm{C}}$ in $R_{\text {cusp }}$ are the magnifications of the merging triplet images. The signs of the magnifications indicate image parities. As the opening angle, namely $\Delta \phi$ in the cusp configuration (the opening angle between images $\mathrm{A}$ and C) and $\phi_{1}$ in the fold configuration (the opening angle between images $\mathrm{A}$ and $\mathrm{B}$ ), approaches zero, $R_{\text {fold }}$ and $R_{\text {cusp }}$ also approach zero when the lensing mass distribution is smooth and the background object is a point source. The anomaly strength of each lens system can therefore be expressed by its $R_{\text {fold }}$ and $R_{\text {cusp }}$.

In order to reduce computational time for the ray-tracing experiment, we randomly draw source positions within a fixed distance from the tangential critical curve where the cusp and fold images form. This distance is fixed to a certain fraction of $r_{\text {caus }}$, where $r_{\text {caus }} \equiv \sqrt{a b}$, and $a$ and $b$ are half the lengths of the long and short axes of the tangential caustic, respectively. After testing different fractions of $r_{\text {caus }}$ on the flux anomaly strength distribution, we select $0.25 r_{\text {caus }}$ for the full ray-tracing run on the mock lenses (see the appendix for more discussion).

\subsection{Ray tracing}

We proceed by running a full ray tracing for the selected simulated galaxies, in order to explore the baryonic effects on flux-ratio anomalies.

The ray tracing is done with two mock lens sets. From each mock lens, 100 sources are randomly sampled inside the main tangential caustic, within the area chosen so that the distance to the tangential caustic is smaller than $0.25 r_{\text {caus }}$, in order to get fold and cusp image configurations. We carry out our calculations in two steps. At the first step we use the ray-tracing code GLAMER (Metcalf \& Petkova 2014) to create a set of idealistic smooth mass models with finite sources. This set of mock lenses provides us with the flux-ratio distributions due to a smooth underlying potential with no contribution from either substructure or baryonic components. These mock lenses are generated using the properties of 15 out of the 50 elliptical galaxies in our Illustris ray-tracing samples. In particular, for each selected lens its total mass, velocity dispersion, and axis ratio are used to generate a particle ensemble for a singular isothermal ellipsoid (SIE) halo that has the same mass resolution as the Illustris-1 simulation.

GLAMER calculates the deflection angles, shear, and convergence by the TREE algorithm (Barnes \& Hut 1986), representing each simulation particle with a B-spline in three dimensions as is commonly done in smooth particle hydrodynamics (SPH) simulations. The size of the particles is set to the distance to the $N_{\text {smooth }}$ th nearest neighbour where $N_{\text {smooth }}$ can be adjusted. This smoothing scheme provides higher resolution where the particles are dense and it is justified, and lower resolution where the particles are sparse and shot noise would otherwise be a problem. GLAMER also has an adaptive ray-shooting capacity to efficiently resolve small images and facilities for finding caustic curves and representing sources of different kinds.

Since GLAMER uses the actual particle distribution for the ray tracing, without fitting the mass distribution with an analytical profile. This feature allows us to quantify the effect of irregular features in the mass distribution, but it also requires some caution in relation to the particle noise (Xu et al. 2009; Rau, Vegetti \& White 2013): on the one hand some degree of smoothing is necessary to avoid the particle noise from contributing significantly to the flux-ratio anomalies, on the other hand, one has to be careful as not to smooth out physical small-scale structures. We smooth the particle distribution, choosing for each particle a smoothing length that depends on its number of neighbours; after testing the effect of different smoothing lengths we chose $N_{\text {smooth }}=64$, which corresponds to $\sim 1-3 \mathrm{kpc}$ near the Einstein radius. Since the characteristic height of a spiral disc is roughly at this physical scale, further increasing the smoothing scale may wash out the edge-on disc structure (see the appendix for more discussion of the particle shot noise.) To properly generate the mock quasar lens images, we use a Gaussian source with $\sigma_{\mathrm{s}}=40 \mathrm{pc}$, based on typical sizes of narrow-line regions (1-100 pc; Moustakas \& Metcalf 2003).

At the second step we go through the same ray-tracing process on the simulated galaxies that we selected from the Illustris simulation. We extract the list of particle positions and masses for each of the selected haloes, selecting only the particles belonging to the main halo. Because of the mass resolution of the Illustris simulation (self-gravitationally bound) subhaloes are only well resolved down to $10^{9} \mathrm{M}_{\odot}(\sim 500$ dark matter particles), which only covers the relatively high mass end of the subhalo mass function. Indeed as shown in $\mathrm{Xu}$ et al. (2009, 2015), systems with anomalous flux ratios are most sensitive to $\mathrm{CDM}$ substructures within a mass range of $10^{7-9} \mathrm{M}_{\odot}$ that are projected in the central few kpc region of the main halo. ${ }^{2}$ As the Illustris simulation does not provide a subhalo population down to this crucial mass range and since we are only interested in quantifying the effect of baryonic structures, we do not include any simulated subhaloes in our ray-tracing analysis.

After the ray-tracing process, the mock lens imaging is convolved with a 50 milliarcsecond (mas) Gaussian point spread function (PSF), which is typical for the beam size for Multi-Element RadioLinked Interferometer Network (MERLIN) observations obtained at $5 \mathrm{GHz}$, to obtain realistic flux ratios. Fig. 4 shows an example of a simulated lens generated by GLAMER after the PSF convolution, in which the lensed images are shown with black contours.

\subsection{Flux-ratio anomaly probability distribution}

Fig. 5 presents the $R_{\text {cusp }}$ and $R_{\text {fold }}$ distributions that were obtained from ray tracing through the smooth mass distributions. The red

\footnotetext{
${ }^{2}$ The upper mass cut is due to the fact that more massive substructures can be ripped apart by tidal interactions and rarely survive in the central region (see figs 14 and 15 of $\mathrm{Xu}$ et al. 2009). The lower mass cut is due to the fact that the perturbation cross-section increases with subhalo mass (see section 5 of Xu et al. 2009 and section 4.5 of Xu et al. 2015 for a detailed discussion)
} 


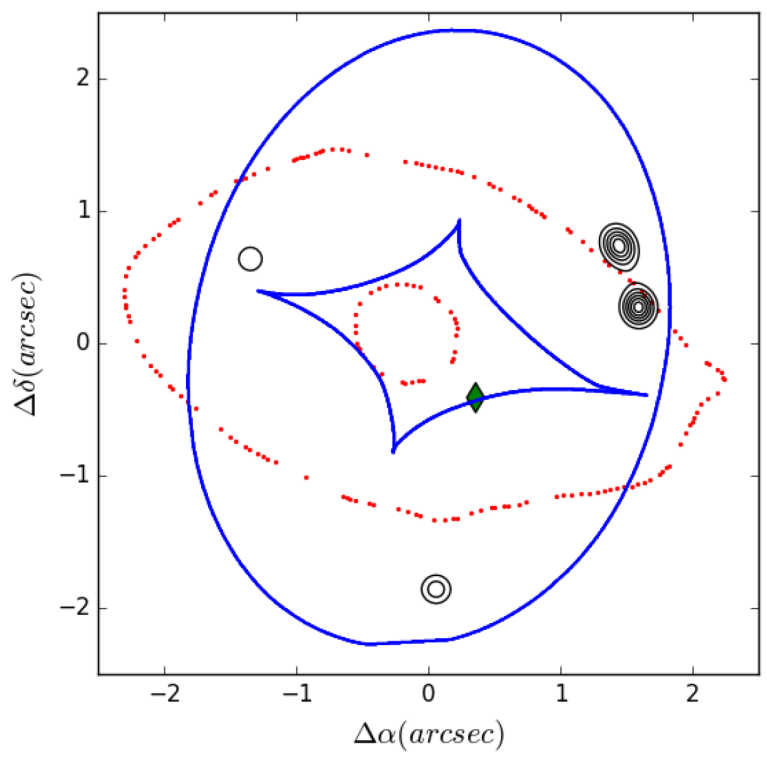

Figure 4. An example of a mock lens showing a typical fold configuration from GLAMER with a $\sigma_{\mathrm{s}}=40 \mathrm{pc}$ Gaussian source and a 50 mas PSF convolution. The lensed images are shown as black contours. The critical curves are shown as red dotted curves, and the caustics are shown as blue solid curves. The source position is marked by the green diamond.

crosses represent the ray-tracing results, with each cross corresponding to one halo-source pair. The curves in each plot are contours, showing the probabilities of obtaining a given value of $R_{\text {cusp }}$ or $R_{\text {fold }}$ or larger, as a function of opening angle. In order to construct the probability contours, the ray-tracing data points are binned every $10^{\circ}$ in $\Delta \phi$ and every $5^{\circ}$ in $\phi_{1}$. These probability distributions are marginally consistent with the smooth results from $\mathrm{Xu}$ et al. (2015, see their fig. 4), where the general smooth lens potentials were modelled as SIEs with observation-motived axis ratios and higher order multipole perturbations plus random external shear. At small opening angles, our ray-tracing results have slightly higher anomaly strengths compared to Xu et al. (2015), because our sources have finite sizes rather than being point sources. The observed $R_{\text {cusp }}$ and $R_{\text {fold }}$ values for real lenses are plotted as black triangles and blue diamonds for elliptical lenses and edge-on disc lenses, respectively (see $\mathrm{Xu}$ et al. 2015 table 1 for more observational details).

Figs 6 and 7 show the ray-tracing results for the simulated disc, elliptical, edge-on disc, and face-on disc lenses that include baryons. We find that the scatter in anomaly strength is larger in the simulated galaxies than in the smooth model lenses for the entire angle range. The probability of finding low anomaly values $\left(\left|R_{\text {cusp }}\right|<0.3\right.$ and $\left.\left|R_{\text {fold }}\right|<0.2\right)$ is comparable for the smooth SIE haloes and the simulated elliptical lenses. However, strong anomalies, especially at small opening angles, can only be found when the higher order moments (mainly from baryonic components) are included. The strong flux anomalies produced by baryonic components are seen in both elliptical and disc lenses. Furthermore, we see that, among all of our galaxy samples, the edge-on disc lenses produce the widest scatter in anomaly strength. Fig. 8 summarizes the ray-tracing results in Figs 5-7 showing the distribution of anomaly values for SIE haloes, elliptical lenses, and disc lenses. We find that the strong anomaly events have larger fractions in both elliptical lenses and disc lenses compared to smooth model.

Tables 2 and 3 list the probability of finding the flux-ratio anomaly strength for observed lenses. The numbers in parentheses show the probability ranges based on the $1 \sigma$ uncertainties of the flux-ratio measurements and the Poisson noise from the simulated flux-ratio distributions. Note that B0128+437 and B2045+265 are outside of the range of opening angles that we explored for the cusp configuration, while $\mathrm{B} 1608+656$ is not listed in the fold configuration for the same reason. Our ray-tracing results show that the baryonic components increase the probability of observing high anomaly strength systems without requiring the presence of dark substructures. Again the systems with edge-on discs are most affected by the baryonic effects.

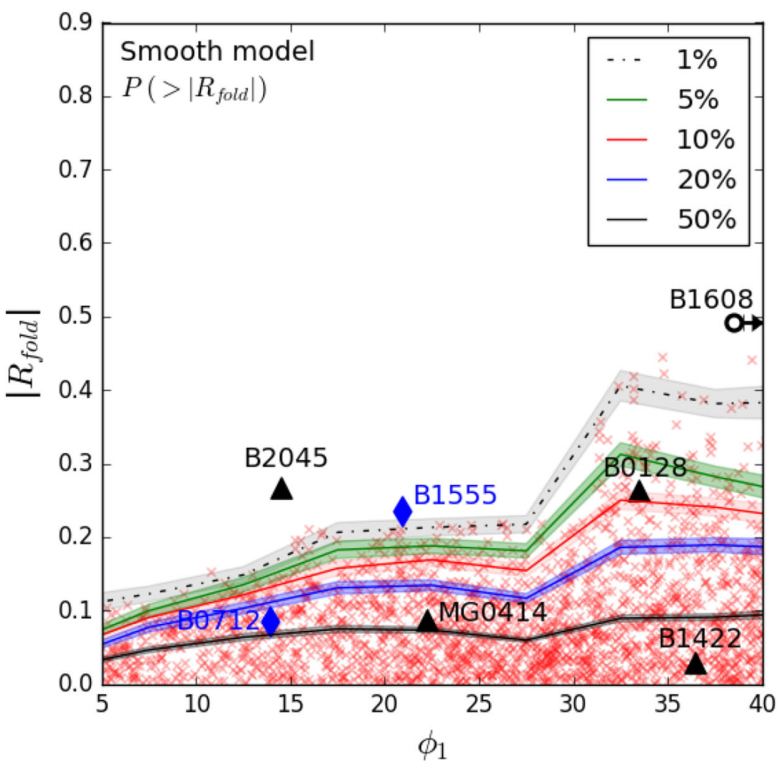

Figure 5. Flux-ratio anomaly strength distributions from the ray tracing of 10 particle haloes that follow the SIE profiles generated by GLAMER. The curves represent $1,5,10,20$, and 50 percent probability of finding $\left|R_{\text {cusp }}\right|$ and $\left|R_{\text {fold }}\right|$ larger than a given value for a given opening angle, while the shaded areas represent $1 \sigma$ uncertainties. The ray-traced data points are shown as red crosses and the observed data points are shown either as blue diamonds (known edge-on discs) or as black triangles. Note that B2045, B0128, and B1608 are outside our complete region of sampling and are represented by open circles. 

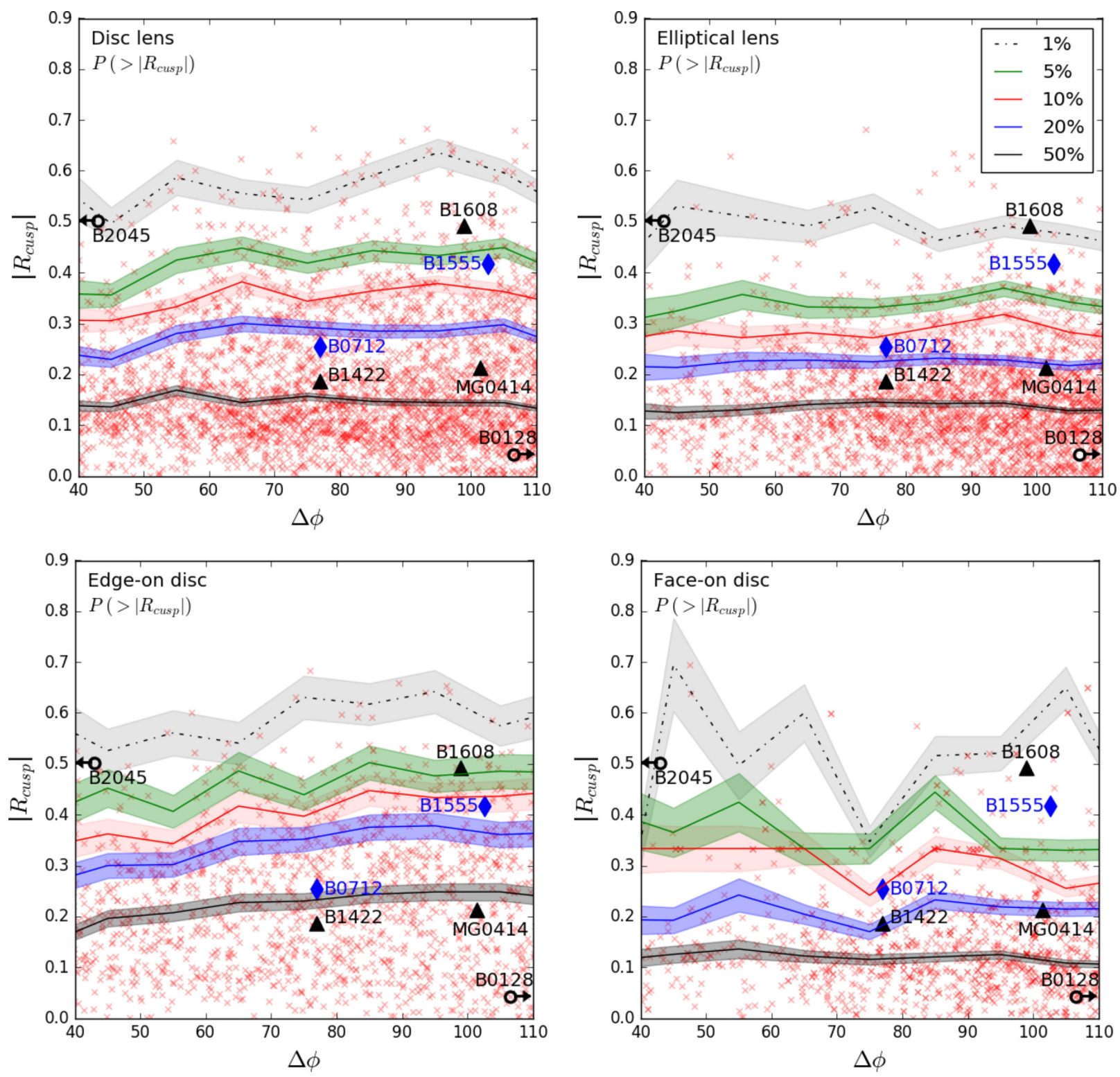

Figure 6. Cusp flux-ratio anomaly strength distribution of four lenses galaxy samples (disc, elliptical, edge-on, and face-on) from the ray-tracing results using GLAMER. The curves represent $1,5,10,20$, and 50 per cent probabilities to find $\left|R_{\text {cusp }}\right|$ larger than a given value for a given opening angle $\left(\Delta \phi\left(^{\circ}\right)\right)$, which the shaded area represents $1 \sigma$ uncertainty. The ray-traced data points are shown as red crosses, the observed data points are shown as black triangles, and the edge-on disc lenses are shown as blue diamonds, respectively. Note that B2045 and B0128 are outside our complete region of sampling and labelled as open circles.

\section{DISCUSSION}

\subsection{Lens samples from Illustris hydrodynamical simulations}

Interpretation of the simulated galaxies is crucial in studies that try to reproduce observed properties. The Illustris project provides a large sample of galaxies with its large-volume hydrodynamical simulation; together with a few other comparable simulations, such as the Evolution and Assembly of GaLaxies and their Environments (EAGLE; Schaye et al. 2015) simulation, it constitutes the state-ofthe-art of hydrodynamical simulations, in terms of resolution and complexity of the baryonic physics implementation. Bottrell et al. (2017) compare the galaxies in the Illustris simulation with the SDSS and found that the galaxies in Illustris are larger in size and have a higher fraction of disc-dominated systems than the samples in SDSS with comparable stellar mass. Vogelsberger et al. (2014a,b) and Genel et al. (2015) also point out that the Illustris simulation only roughly reproduces the stellar mass function and the morphology distributions. We will discuss how these differences limit our sample selection strategy and the interpretation of our analysis results.

In general, the Illustris simulation has stronger feedback processes than other similar simulations - for example the EAGLE simulation (Vogelsberger et al. 2014a; Schaye et al. 2015; Despali $\&$ Vegetti 2017). The only concern is if the Illustris simulation can provide us a realistic selection of galaxies containing all morphological types. In the Illustris simulation, there are higher fraction of disc systems and also higher fraction of disc-dominated systems among the disc galaxies. Therefore, instead of selecting a galaxy sample with all morphological types, we select only the two extreme 

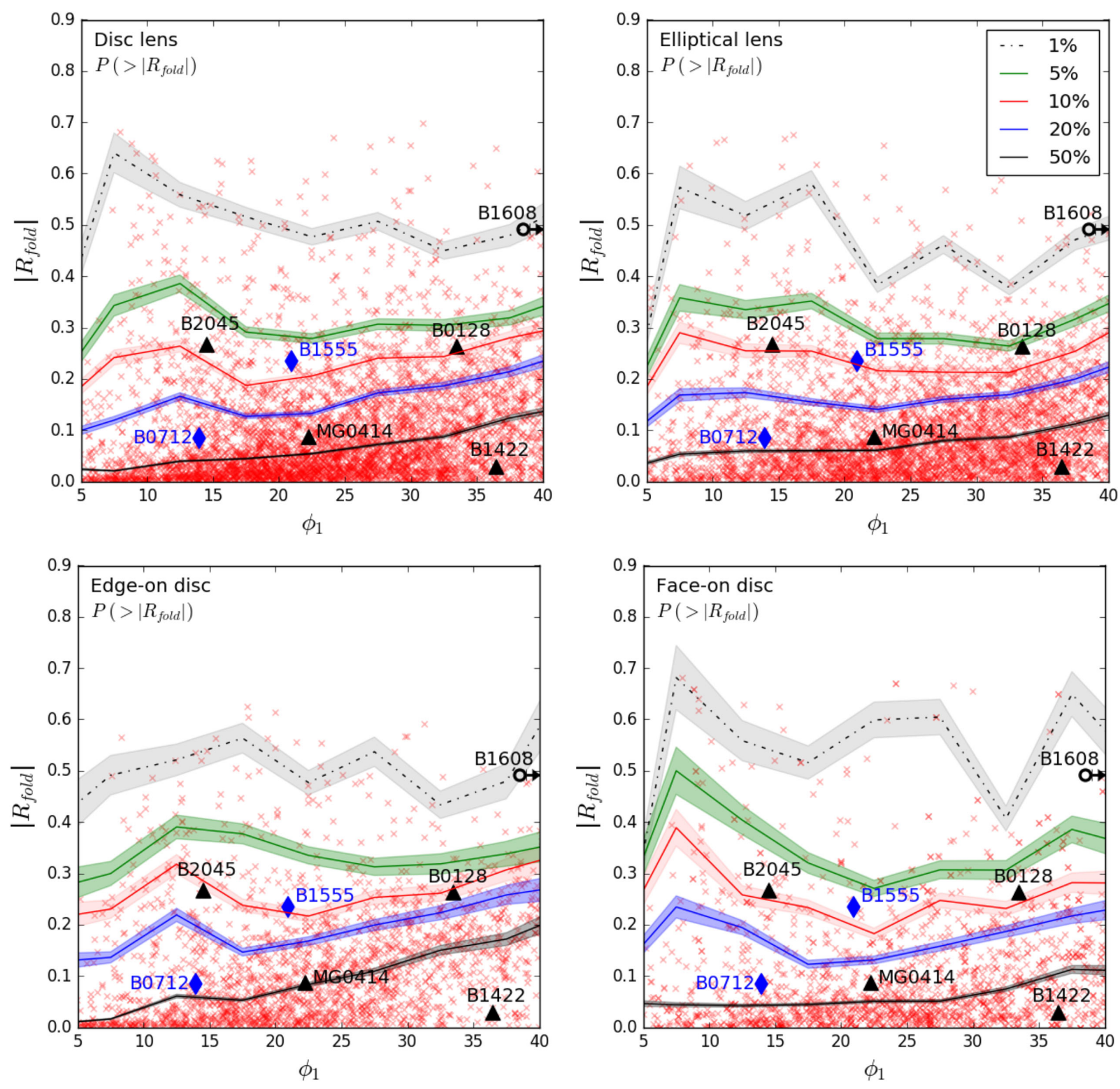

Figure 7. Fold flux-ratio anomaly strength distribution of four lenses galaxy samples (disc, elliptical, edge-on, and face-on) from the ray-tracing results using GLAMER. The curves represent $1,5,10,20$, and 50 per cent probabilities to find $\left|R_{\text {fold }}\right|$ larger than a given value for a given opening angle $\left(\phi_{1}\left({ }^{\circ}\right)\right)$, which the shaded area represents $1 \sigma$ uncertainty. The ray-traced data points are shown as red crosses, the observed data points are shown as black triangles, and the edge-on disc lenses are shown as blue diamonds, respectively. Note that B1608 is outside our complete region of sampling and labelled as an open circle.

categories on the morphological spectrum: massive ellipticals without signs of disc-like structures and late-type disc galaxies with well-developed thin discs. Also, by introducing the kinematic criteria into our selection process, our classification of elliptical and disc galaxies has a better performance than that obtained by using the photometric criteria alone. This is supported by Bottrell et al. (2017), who showed that the simulated galaxy sample has a better agreement with the observed galaxy sample once the internal kinematics come into the analysis.

In this paper, we focus on the baryon-induced flux-ratio anomalies and compare the strength of the anomalies between elliptical and disc lenses. Although biases in the Illustris simulation may limit our ability to construct a realistic mock survey sample, our data are still adequate for investigating the baryonic effects in the two categories of lens galaxies with the lowest and the strongest perturbation ability.

\subsection{Baryonic effects on flux-ratio anomalies}

The idea that baryonic components such as edge-on discs can generate perturbations to the lensing potential similar to those produced by dark substructures and, therefore, can cause flux-ratio anomalies in lensed quasars has been supported by previous investigations of the B1555+375 and B0712+472 systems (Hsueh et al. 2016, 2017). Additionally, Gilman et al. (2017) have shown that the luminous structure in elliptical lenses may account for $10-15$ per cent of flux-ratio anomalies. In this work, we select galaxies in the Illustris simulation with well-developed disc structure and compare them to typical elliptical lenses. By comparing the ray-tracing results of simulated galaxies that include baryons with those from the smooth SIE haloes, we find clear signatures of flux-ratio anomalies that have been produced by the higher order moments that are mainly originated from baryonic structure (see Figs 5-7). The scatter in the 

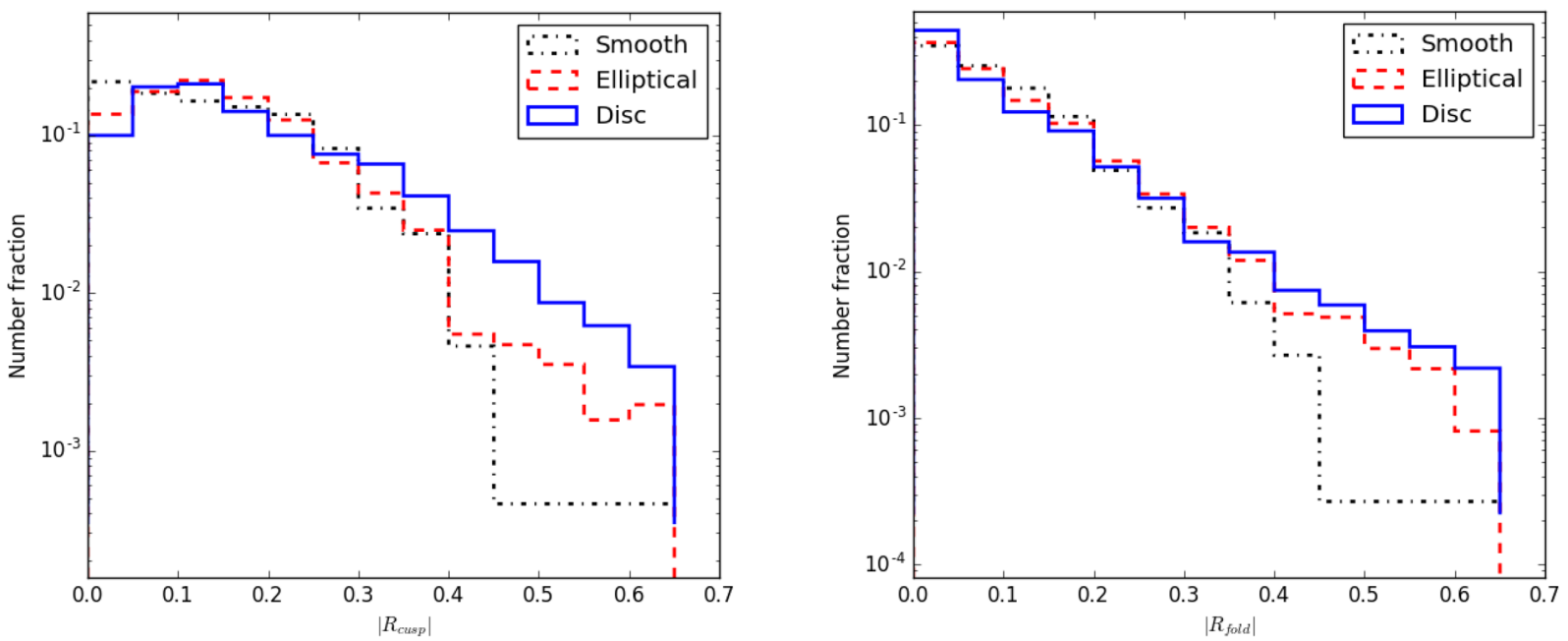

Figure 8. Number fraction histograms showing the distribution of on flux-ratio anomaly strength $R_{\text {cusp }}$ (left) and $R_{\text {fold }}$ (right) from the ray-tracing results, which black dotted lines represent smooth model, red solid lines represent elliptical lenses, and the blue solid lines represent disc lenses, respectively. Strong anomaly events are more likely to be found with the presence of baryons compared to the smooth model lenses.

Table 2. Probability of cusp configuration anomaly strength in observed lenses, sorted by $\left|R_{\text {cusp }}\right|$. The numbers in parentheses are the probabilities from the $1 \sigma$ uncertainty from flux-ratio measurements and Poisson noise of ray-tracing data points. B0128+437 is not listed because its large opening angle $\left(\Delta \phi=123^{\circ}\right)$ is out of our sampling region. B2045+265 is similarly not listed due to its small opening angle $\left(\Delta \phi=35^{\circ}\right)$. Note that B0712+472 and B1555+375 are confirmed edge-on disc lenses.

\begin{tabular}{lccccccc}
\hline Lens & $\left|R_{\text {cusp }}\right|$ & $\Delta \phi\left(^{\circ}\right)$ & $P_{\text {smooth }}\left(>\left|R_{\text {cusp }}\right|\right)$ & $P_{\text {ell }}\left(>\left|R_{\text {cusp }}\right|\right)$ & $P_{\text {disc }}\left(>\left|R_{\text {cusp }}\right|\right)$ & $P_{\text {edge }}\left(>\left|R_{\text {cusp }}\right|\right)$ & $P_{\text {face }}\left(>\left|R_{\text {cusp }}\right|\right)$ \\
\hline B1422+231 & 0.187 & 77.0 & $0.36(0.26,0.46)$ & $0.27(0.20,0.34)$ & $0.37(0.31,0.43)$ & $0.61(0.54,0.69)$ & $0.17(0.11,0.22)$ \\
MG 0414+0534 & 0.213 & 101.5 & $0.31(0.10,0.52)$ & $0.22(0.11,0.44)$ & $0.33(0.22,0.49)$ & $0.57(0.45,0.68)$ & $0.17(0.08,0.31)$ \\
B0712+472 & 0.254 & 76.9 & $0.12(0.08,0.21)$ & $0.12(0.06,0.19)$ & $0.24(0.18,0.29)$ & $0.43(0.37,0.50)$ & $0.10(0.03,0.17)$ \\
B1555+375 & 0.417 & 102.6 & $0.01(0.00,0.07)$ & $0.02(0.00,0.07)$ & $0.07(0.05,0.09)$ & $0.13(0.05,0.20)$ & $0.02(0.00,0.07)$ \\
B1608+656 & 0.492 & 99.0 & $0.00(0.00,0.08)$ & $0.01(0.00,0.06)$ & $0.03(0.00,0.13)$ & $0.05(0.01,0.09)$ & $0.01(0.00,0.04)$ \\
\hline
\end{tabular}

Table 3. Probability of fold configuration anomaly strength in observed lenses, sorted by $\left|R_{\text {fold }}\right|$. The numbers in parentheses are the probabilities from the $1 \sigma$ uncertainty from flux-ratio measurements and Poisson noise of ray-tracing data points. B1608+656 is not listed because its large opening angle $\left(\phi_{1}=48^{\circ}\right)$ is out of our sampling region. Note that B0712+472 and B1555+375 are confirmed edge-on disc lenses.

\begin{tabular}{lccccccc}
\hline Lens & $\left|R_{\text {fold }}\right|$ & $\phi_{1}\left(^{\circ}\right)$ & $P_{\text {smooth }}\left(>\left|R_{\text {fold }}\right|\right)$ & $P_{\text {ell }}\left(>\left|R_{\text {fold }}\right|\right)$ & $P_{\text {disc }}\left(>\left|R_{\text {fold }}\right|\right)$ & $P_{\text {edge }}\left(>\left|R_{\text {fold }}\right|\right)$ & $P_{\text {face }}\left(>\left|R_{\text {fold }}\right|\right)$ \\
\hline B1422+231 & 0.03 & 36.4 & $0.82(0.79,0.85)$ & $0.85(0.82,0.88)$ & $0.84(0.82,0.87)$ & $0.95(0.88,0.95)$ & $0.82(0.80,0.85)$ \\
B0712+472 & 0.085 & 13.9 & $0.44(0.30,0.60)$ & $0.40(0.33,0.50)$ & $0.31(0.26,0.39)$ & $0.39(0.32,0.47)$ & $0.33(0.28,0.41)$ \\
MG 0414+0534 & 0.087 & 22.2 & $0.45(0.22,0.71)$ & $0.35(0.22,0.65)$ & $0.34(0.20,0.60)$ & $0.49(0.30,0.71)$ & $0.31(0.19,0.59)$ \\
B1555+375 & 0.235 & 20.9 & $0.01(0.00,0.07)$ & $0.09(0.05,0.14)$ & $0.07(0.05,0.12)$ & $0.10(0.04,0.17)$ & $0.08(0.05,0.17)$ \\
B0128+437 & 0.263 & 33.5 & $0.11(0.07,0.15)$ & $0.05(0.01,0.12)$ & $0.09(0.05,0.16)$ & $0.12(0.05,0.21)$ & $0.10(0.05,0.20)$ \\
B2045+265 & 0.267 & 14.5 & $0.00(0.00,0.06)$ & $0.10(0.05,0.18)$ & $0.08(0.05,0.15)$ & $0.11(0.05,0.19)$ & $0.09(0.05,0.20)$ \\
\hline
\end{tabular}

ray-tracing results (red crosses in Figs 5-7) indicate that the baryons produce more systems that have strong anomaly strengths (i.e. large values of $R_{\text {cusp }}$ and $R_{\text {fold }}$ ), and therefore increase the probability of finding such systems. This increase in the strength of the anomalies compared to the smooth model results in Fig. 5 can be clearly seen in the shift of the 5 per cent probability contours, and even, for the disc lenses, of the 20 per cent contours.

Events in strong anomaly regions (i.e. $\left|R_{\text {cusp }}\right|>0.3$ and $\left.\left|R_{\text {fold }}\right|>0.2\right)$ are particularly rare in the smooth model distributions. By comparing the ray-tracing results in Figs 5-7, we find that baryons increase the probability of these extreme events by about 8 per cent in elliptical lenses but produce no significant boost in low anomaly regions. For disc lenses, these shifts in the probability curves to higher anomaly values happen on all scales and are most significant at small opening angles. Fig. 8 shows the number fraction distribution of anomaly strengths, which demonstrates the difference between the smooth models and simulated lenses with baryons more clearly. The fraction of elliptical lenses and disc lenses exceed the fraction of SIE haloes in strong anomaly regions, and the disc lenses show significantly higher fractions in the most extreme events in $\left|R_{\text {cusp }}\right|$. These trends are also observed in Tables 2 and 3, showing the probabilities of finding observed lenses among particle haloes generated from smooth mass models and mock lenses of different morphological types from the simulation. It is also interesting to notice that the behaviour of baryon-induced anomalies is different from that of substructure-induced anomalies. The former are seen for cusp/fold images with a large range of opening angles, while the latter are only significant at very small opening angles (see fig. 4 of $\mathrm{Xu}$ et al. 2015). This indicates that, in the large opening angle systems, the flux-ratio anomalies are more likely to be baryon 
induced than substructure induced, which provides a hint of a method for distinguishing the major sources of flux anomaly in radio-loud lenses.

While current estimates of substructure abundance and substructure detection using flux-ratio anomalous lenses are based on the assumption that the observed anomalies are generated solely by substructures (Bradač et al. 2002; Dalal \& Kochanek 2002; Fadely $\&$ Keeton 2012; Nierenberg et al. 2014), we find that the baryonic components can also provide a major contribution to flux-ratio anomalies. Because of the mass resolution of the simulation, we cannot include realistic substructures in this work. However, our results still provide an explanation for the results seen in $\mathrm{Xu}$ et al. (2015), in which they found that dark matter substructures alone are not sufficient to reproduce the observed flux anomaly strengths. Among the four systems that have only a few per cent probability for their flux ratios to be solely attributed to the presence of cold dark matter substructures, including baryons makes the observed strong flux anomalies in B0712+472, B1422+231, and B1555+375 more likely; while B2045+265 still has a low probability even in the presence of baryonic components. (However, its high anomaly may be explained by a luminous satellite, see McKean et al. 2007, for more discussion.)

\subsection{The effect of disc inclination angle}

In recent investigations of two confirmed edge-on disc lenses, B0712+472 and B1555+375, Hsueh et al. $(2016,2017)$ show that the inclusion of exponential disc components in the lens model is sufficient to reproduce the observed flux-ratio anomalies without any need to invoke dark matter substructure. Those results have motivated the work presented in this paper. We have found that our general disc lens sample, which includes a range of inclination angles, has a higher probability of causing high flux anomaly systems than the elliptical lens sample. However, we expect that the disc inclination angle can be an important parameter when considering the effects of baryons in lens systems. This is borne out by the ray tracing. As seen in Figs 6 and 7, the edge-on discs show larger scatter in flux anomaly strength than both the face-on discs and ellipticals, while the face-on disc lenses produce results similar to what is seen in the elliptical lenses. These results indicate that the spiral arms in the face-on disc may not be able to cause strong anomalous flux ratios. Among the morphological types considered here, the edge-on disc lenses produce the strongest flux-ratio anomalies. In Tables 2 and 3, the last four columns indicate that baryons increase the probability of finding systems such as B0712+472 and $\mathrm{B} 1555+375$, with the edge-on disc lens sample showing the largest increase of the probability of producing large anomalies. For example, the probabilities of obtaining the observed flux-ratio anomalies in $\mathrm{B} 1555+375$ and $\mathrm{B} 0712+472$ with smooth mass distributions are only 1 and 10 per cent, respectively. By including edge-on discs, these probabilities rise to $8-15$ and $30-40$ per cent, respectively. In general, the baryonic components increase the probability of finding large flux-ratio anomalies by $\sim 10-20$ per cent for the disc lenses compared to the early-type lenses.

In a few cases of face-on lenses, we found that their spiral arms can also distort the critical curves and cause strong flux-ratio anomalies (see Fig. $\mathrm{C} 1$ and Appendix $\mathrm{C}$ for more discussion). It is also notable that while all the criteria we used to identify morphological types are designed to select typical examples of each type, the real lens systems can exhibit more complex structures. For example, galaxies with a large thick disc are not selected as 'disc' galaxies in this work, but their thick discs can still be a more efficient perturber than the stellar structure in the elliptical galaxies.

In conclusion, our ray-tracing results show that the edge-on disc lenses are the strongest baryonic perturber and have the ability to induce high flux-ratio anomaly in strongly lensed quasars. Their high flux-ratio anomalies can be reproduced by including an exponential disc into the lens model (Hsueh et al. 2016, 2017). These results suggest that the standard 'SIE+shear' model is not an appropriate analytical model for the edge-on disc lenses. In Fig. 2, the histograms show that the disc lenses mostly are less massive and have small Einstein radii or, conversely, the smaller the Einstein radius is, the higher the chance that the lensing galaxy is a disc. However, at these small Einstein radii, it could be difficult to confirm the morphology of the lens galaxy, especially if the lensed quasar images are bright. In the current sample of radio-loud lensed quasars, three out of eight lenses (B0712+472, B1555+375, and $\mathrm{B} 1933+503)$ are confirmed to be disc galaxies, B0128+437 is a small separation system and shows possible disc morphology in the Keck adaptive optics (AO) imaging (Lagattuta, Auger \& Fassnacht 2010), B1608+656 is a galaxy merger with complicated baryonic structures close to the lensed images, and the rest of them show no obvious sign of discs. Therefore, at least half of the current sample probably requires the consideration of baryonic effects. Moreover, both Gilman et al. (2017) and this current work also show that there is evidence of baryon-induced flux anomalies in elliptical lenses. This raises the possibility of a bias in the analysis of lens flux ratios, introduced by the lack of knowledge about the baryonic structures in the lens galaxies. It is also notable that disc lenses tend to dominate among small angular separation systems (e.g. Turner et al. 1984), which is also shown in Fig. 2. As the next generation of large-scale surveys, such as the Dark Energy Survey (Bechtol et al. 2015), Euclid (Cimatti \& Scaramella 2012), and the Large Synoptic Survey Telescope (LSST Dark Energy Science Collaboration 2012) will be more efficient in finding small angular separation systems, we expect the disc system fraction to increase compared to the current sample.

\subsection{Astrometric anomalies in the Illustris galaxies}

The work of Gilman et al. (2017), in which mock lenses included the gravitational perturbation from the luminous mass distribution, shows that the baryonic components can generate flux-ratio anomalies but not astrometric anomalies. With our ray-tracing results from the simulated galaxies, we are also able to explore the astrometric anomalies generated by the baryonic components. The astrometric anomaly is defined to be the absolute sum of the deviation between the 'real' image positions of the mock lenses and their corresponding best-fitting positions produced by a SIE plus external shear model. We analyse the elliptical and edge-on disc mock lenses to see if the morphology of the lens galaxy can also affect astrometric anomalies. We find that about 13 percent of smooth model predictions have astrometric anomalies larger than 3 mas, which is the comparable to the precision obtained by very long baseline radio interferometric observations. No obvious difference between the elliptical and edge-on disc lenses in their astrometric anomalies is seen. Our results indicate that the idea of identifying baryonic perturbations by the lack of an observed astrometric anomaly may need to be revisited, since we have seen that baryonic components can also be a cause of astrometric anomalies. 


\section{CONCLUSION}

Motivated by the latest studies on edge-on disc lenses and the resulting baryonic effects on flux-ratio anomalies, we present the first statistical investigation of the baryonic contribution to flux-ratio anomalies from a numerical simulation perspective. Our ray-tracing results support the idea that the presence of baryonic structures can induce flux-ratio anomalies (see Figs 5-7), which are clearly seen in both disc lenses and elliptical lenses. Edge-on disc lenses produced the strongest anomalies among the morphological types that we studied. These results offer a possible explanation for why the observed flux anomaly frequency is higher than what is expected from CDM subhaloes as predicted by state-of-the-art $\mathrm{N}$-body simulations (Xu et al. 2009, 2015). While the magnitudes of the shifts in probability due to the presence of baryons may differ depending on which simulation is used to explore the effect, we expect that the qualitative result of having the baryons increase the likelihood of high values of $R_{\text {cusp }}$ and $R_{\text {fold }}$ is a generic phenomenon. More detailed investigations can be done on predicting baryonic effects in mock surveys when future hydrodynamical simulations are able to construct more realistic galaxy populations.

As the monitoring of lensed quasars brings the uncertainties on observed flux ratios down to 5 percent (Koopmans et al. 2003), properly modelling the lens galaxy becomes an increasingly important aspect in the use of flux ratios to provide inferences on substructure abundance. Our results show that the inclusion of baryonic effects in the modelling is a critical step in this process, especially in the case of disc lenses. The morphology of the lensing galaxy also needs to be considered in terms of the dark matter substructure where, once again, the presence of a disc can have an important effect. Studies show that interactions between the disc and substructures can destroy the clumps and cause a lower substructure abundance in disc galaxies compared to haloes with elliptical galaxies (D’Onghia et al. 2010; Yurin \& Springel 2015; Zhu et al. 2016; Errani et al. 2017). Because of the mass resolution in the Illustris simulation, we cannot include realistic subgalactic substructures in this work. However, future higher resolution simulations can be used to investigate the contribution of both substructures and baryons to flux-ratio anomalies in the future.

\section{ACKNOWLEDGEMENTS}

J-WH and CDF would like to thank the Max Planck Institute for Astrophysics for the warm hospitality during their visits, and J-WH additionally thanks the Heidelberg Institute for Theoretical Studies (HITS) for kindly hosting her visit. CDF acknowledges support from the US National Science foundation grant AST-1312329. DX would like to thank the Klaus Tschira Foundation.

\section{REFERENCES}

Abadi M. G., Navarro J. F., Steinmetz M., Eke V. R., 2003, ApJ, 597, 21 Barnes J., Hut P., 1986, Nature, 324, 446

Bechtol K. et al., 2015, ApJ, 807, 50

Blandford R., Narayan R., 1986, ApJ, 310, 568

Bottrell C., Torrey P., Simard L., Ellison S. L., 2017, MNRAS, 467, 2879

Bradač M., Schneider P., Steinmetz M., Lombardi M., King L. J., Porcas R., 2002, A\&A, 388, 373

Browne I. W. A., Wilkinson P. N., Patnaik A. R., Wrobel J. M., 1998, MNRAS, 293, 257

Browne I. W. A. et al., 2003, MNRAS, 341, 13

Chen J., Koushiappas S. M., Zentner A. R., 2011, ApJ, 741, 117
Chiba M., Minezaki T., Kashikawa N., Kataza H., Inoue K. T., 2005, ApJ, 627,53

Cimatti A., Scaramella R., 2012, Mem. Soc. Astron. Ital. Suppl., 19, 314

Dalal N., Kochanek C. S., 2002, ApJ, 572, 25

Despali G., Vegetti S., 2017, MNRAS, 469, 1997

Dobler G., Keeton C. R., 2006, MNRAS, 365, 1243

D’Onghia E., Vogelsberger M., Faucher-Giguere C.-A., Hernquist L., 2010, ApJ, 725, 353

Errani R., Peñarrubia J., Laporte C. F. P., Gómez F. A., 2017, MNRAS, 465, L59

Fadely R., Keeton C. R., 2012, MNRAS, 419, 936

Genel S. et al., 2014, MNRAS, 445, 175

Genel S., Fall S. M., Hernquist L., Vogelsberger M., Snyder G. F., RodriguezGomez V., Sijacki D., Springel V., 2015, ApJ, 804, L40

Gilman D., Agnello A., Treu T., Keeton C. R., Nierenberg A. M., 2017, MNRAS, 467, 3970

Hezaveh Y. D. et al., 2016, ApJ, 823, 37

Hinshaw G. et al., 2013, ApJS, 208, 19

Hsueh J.-W., Fassnacht C. D., Vegetti S., McKean J. P., Spingola C., Auger M. W., Koopmans L. V. E., Lagattuta D. J., 2016, MNRAS, 463, L51

Hsueh J.-W. et al., 2017, MNRAS, 469, 3713

Keeton C. R., 2001, preprint (arXiv:astro-ph/0102341)

Kochanek C. S., Dalal N., 2004, ApJ, 610, 69

Koopmans L. V. E., 2005, MNRAS, 363, 1136

Koopmans L. V. E., de Bruyn A. G., 2000, A\&A, 358, 793

Koopmans L. V. E. et al., 2003, ApJ, 595, 712

Lagattuta D. J., Auger M. W., Fassnacht C. D., 2010, ApJ, 716, L185

LSST Dark Energy Science Collaboration, 2012, preprint (arXiv:1211.0310)

Macciò A. V., Miranda M., 2006, MNRAS, 368, 599

McCully C., Keeton C. R., Wong K. C., Zabludoff A. I., 2014, MNRAS, 443,3631

McKean J. P. et al., 2007, MNRAS, 378, 109

Mao S., 1992, ApJ, 389, L41

Mao S., Schneider P., 1998, MNRAS, 295, 587

Mao S., Jing Y., Ostriker J. P., Weller J., 2004, ApJ, 604, L5

Metcalf R. B., 2005, ApJ, 629, 673

Metcalf R. B., Amara A., 2012, MNRAS, 419, 3414

Metcalf R. B., Madau P., 2001, ApJ, 563, 9

Metcalf R. B., Petkova M., 2014, MNRAS, 445, 1942

Metcalf R. B., Zhao H., 2002, ApJ, 567, L5

Minezaki T., Chiba M., Kashikawa N., Inoue K. T., Kataza H., 2009, ApJ, 697, 610

Mittal R., Porcas R., Wucknitz O., 2007, A\&A, 465, 405

Möller O., Hewett P., Blain A. W., 2003, MNRAS, 345, 1

Moustakas L. A., Metcalf R. B., 2003, MNRAS, 339, 607

Myers S. T. et al., 2003, MNRAS, 341, 1

Nelson D. et al., 2015, Astron. Comput., 13, 12

Nierenberg A. M., Treu T., Wright S. A., Fassnacht C. D., Auger M. W., 2014, MNRAS, 442, 2434

Nierenberg A. M. et al., 2017, MNRAS, 471, 2224

Patnaik A. R., Browne I. W. A., Wilkinson P. N., Wrobel J. M., 1992, MNRAS, 254, 655

Quadri R., Möller O., Natarajan P., 2003, ApJ, 597, 659

Rau S., Vegetti S., White S. D. M., 2013, MNRAS, 430, 2232

Schaye J. et al., 2015, MNRAS, 446, 521

Schneider P., Weiss A., 1992, A\&A, 260, 1

Springel V., 2010, ARA\&A, 48, 391

Teklu A. F., Remus R.-S., Dolag K., Beck A. M., Burkert A., Schmidt A. S., Schulze F., Steinborn L. K., 2015, ApJ, 812, 29

Turner E. L., Ostriker J. P., Gott J. R., III, 1984, ApJ, 284, 1

Vegetti S., Koopmans L. V. E., 2009, MNRAS, 392, 945

Vegetti S., Koopmans L. V. E., Bolton A., Treu T., Gavazzi R., 2010 , MNRAS, 408, 1969

Vegetti S., Lagattuta D. J., McKean J. P., Auger M. W., Fassnacht C. D., Koopmans L. V. E., 2012, Nature, 481, 341

Vegetti S., Koopmans L. V. E., Auger M. W., Treu T., Bolton A. S., 2014, MNRAS, 442, 2017 
Vogelsberger M., Genel S., Sijacki D., Torrey P., Springel V., Hernquist L., 2013, MNRAS, 436, 3031

Vogelsberger M. et al., 2014a, MNRAS, 444, 1518

Vogelsberger M. et al., 2014b, Nature, 509, 177

Wilkinson P. N., Browne I. W. A., Patnaik A. R., Wrobel J. M., Sorathia B., 1998, MNRAS, 300, 790

Winn J. N., Rusin D., Kochanek C. S., 2004, Nature, 427, 613

Xu D. D. et al., 2009, MNRAS, 398, 1235

Xu D. D., Mao S., Cooper A. P., Wang J., Gao L., Frenk C. S., Springel V., 2010, MNRAS, 408, 1721

Xu D. D., Mao S., Cooper A. P., Gao L., Frenk C. S., Angulo R. E., Helly J., 2012, MNRAS, 421, 2553

Xu D., Sluse D., Gao L., Wang J., Frenk C., Mao S., Schneider P., Springel V., 2015, MNRAS, 447, 3189

Xu D., Springel V., Sluse D., Schneider P., Sonnenfeld A., Nelson D., Vogelsberger M., Hernquist L., 2017, MNRAS, 469, 1824

Yurin D., Springel V., 2015, MNRAS, 452, 2367

Zakharov A. F., 1995, A\&A, 293, 1

Zhu Q., Marinacci F., Maji M., Li Y., Springel V., Hernquist L., 2016, MNRAS, 458, 1559
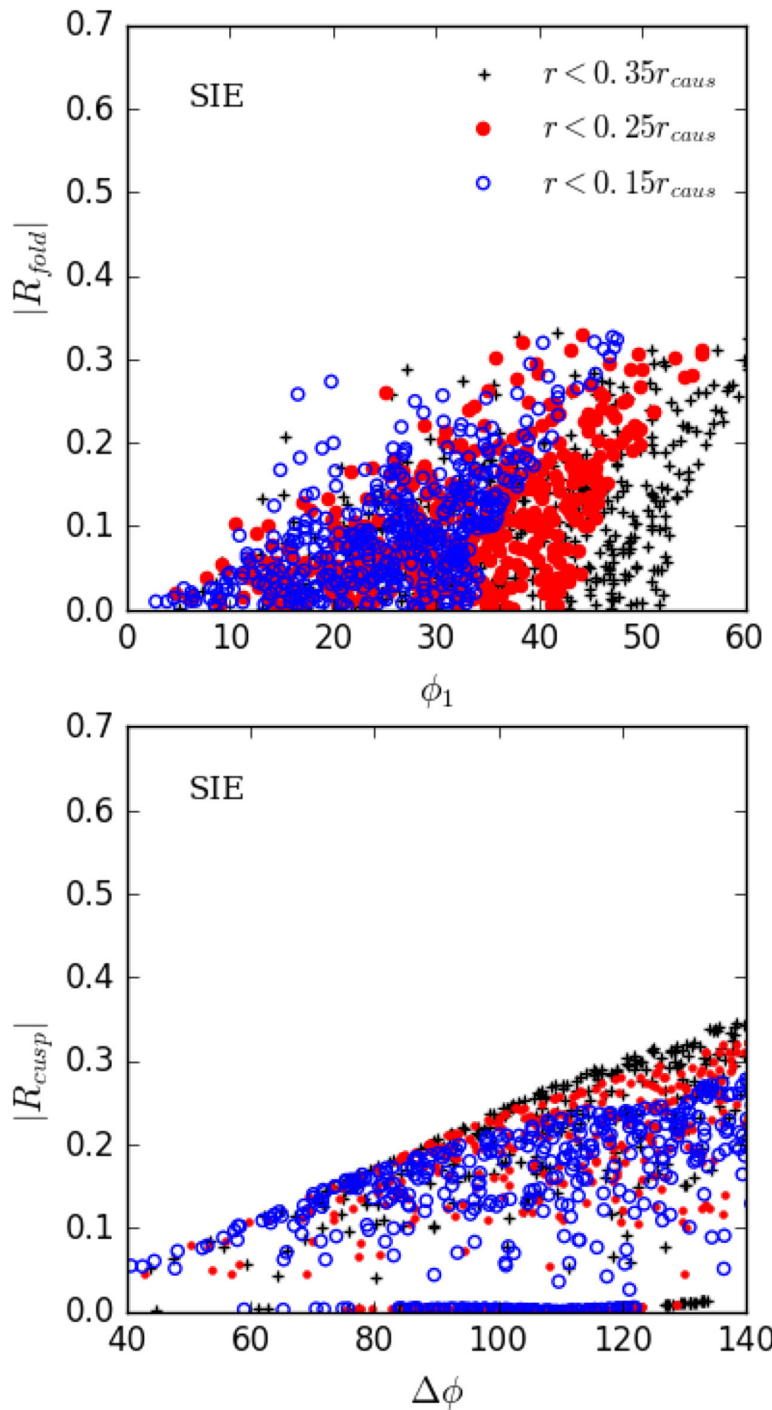

\section{APPENDIX A: TESTING THE SELECTION AREA ON SOURCE PLANE}

To test if our selected fraction of $r_{\text {caus }}$ can reproduce the realistic flux anomaly strength distribution within the opening angle we are interested, we first use the lens modelling code GRAVLENS (Keeton 2001) to create analytical distributions where the source positions are generated using different fractions of $r_{\text {caus }}$. Fig. A1 shows the distributions of $R_{\text {fold }}$ versus $\phi_{1}$ (top panels) and $R_{\text {cusp }}$ versus $\Delta \phi$ (bottom panels) for an elliptical lens (left-hand panels) and an edgeon disc lens (right-hand panels). Open circles, solid circles, and crosses represent the distributions for source positions generated within $0.15 r_{\text {caus }}, 0.25 r_{\text {caus }}$, and $0.35 r_{\text {caus }}$ from the tangential caustic, respectively. As can be seen, the distributions start to diverge beyond certain values of the opening angle, with the divergence occurring at smaller angles for smaller fractions of $r_{\text {caus }}$. For example, for the $0.25 r_{\text {caus }}$, the divergence from the $0.35 r_{\text {caus }}$ distributions occur at $\phi_{1}=45^{\circ}$ in the $R_{\text {fold }}-\phi_{1}$ plane, while in the $R_{\text {cusp }}-\Delta \phi$ plane the
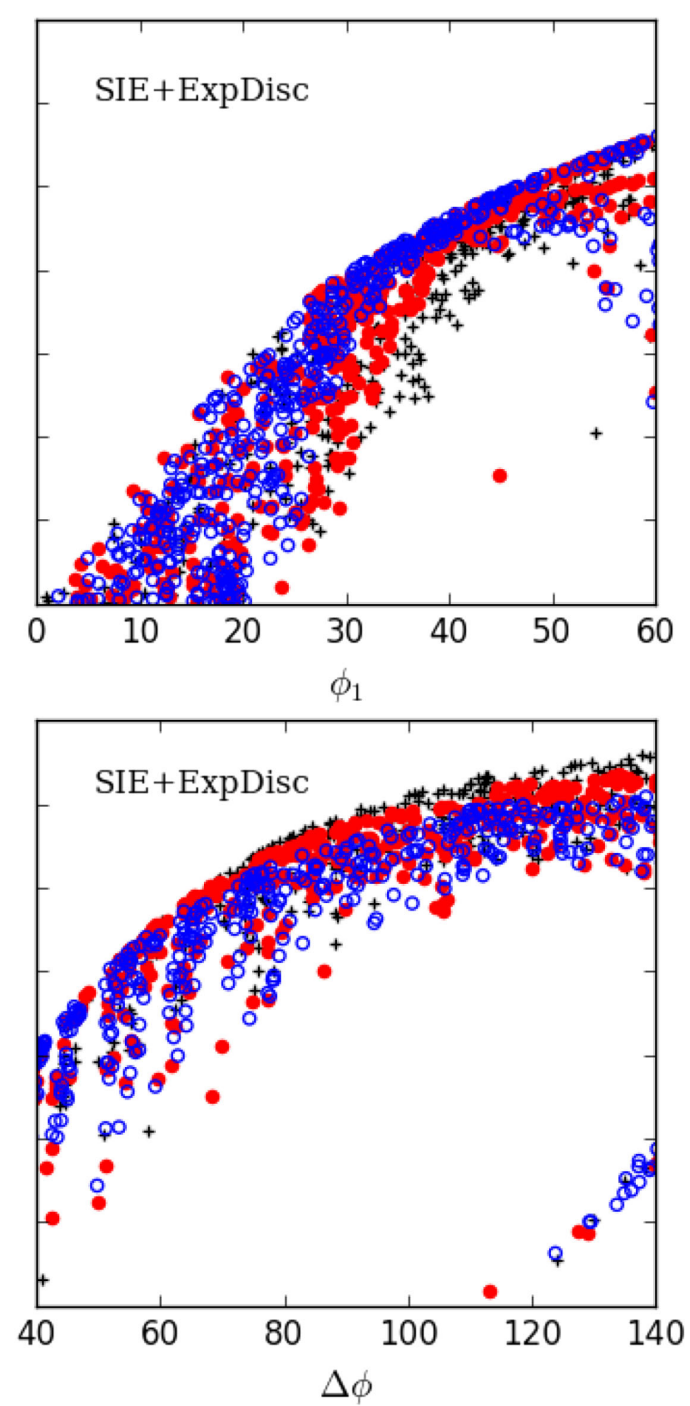

Figure A1. Completeness test of flux-ratio anomalies on the analytical model of an elliptical lens and a disc lens, generated by GRAVLENS. Left: singular isothermal ellipsoid (SIE) model with ellipticity $e=0.38$. Right: SIE plus exponential disc (SIE+Expdisc) model with $e=0.76$ and disc mass fraction within Einstein radius equals 15 per cent. Each data point represents a point source drawn randomly within the tangential caustic which the distance to the tangential caustic is smaller than $0.15 r_{\text {caus }}$ (blue open circles), $0.25 r_{\text {caus }}$ (red circles), and $0.35 r_{\text {caus }}$ (black crosses). Top: absolute value of $R_{\text {fold }}$ and the fold double opening angle $\Delta \phi$. Bottom: absolute value of $R_{\text {cusp }}$ and the cusp triplet opening angle $\phi_{1}$. 
divergence occurs at $\Delta \phi \sim 110^{\circ}$. The majority of the observed flux anomaly lenses (see Section 3.2) have opening angles below these values. We therefore set the source region to be within $0.25 r_{\text {caus }}$ to the tangential caustic for the full ray-tracing run on the simulated lenses.

\section{APPENDIX B: SHOT NOISE TEST}

Shot noise is a well-known problem that one can encounter when ray tracing through simulated haloes (Rau et al. 2013) and it is important to carefully estimate its impact in order to validate our results. Thus, to understand the contribution from particle noise in our ray-tracing results, we take 10 haloes from the Illustris simulation and for each of them, we generate SIE haloes into analytical form and particle ensembles: these last have the same resolution of the original data, but a smooth particle distribution, so that we can test the effect of shot noise in a set-up where there is no irregular structure in the lens halo. We then ray trace through them with the same setting as we described in Section 3.1. Both for the analytical and the particle SIE, we use the shape parameters obtained by fitting a SIE to the original data. Fig. B1 shows that the anomalous strength in analytical SIE and particle SIE (which is the smooth model described in Section 3.1) is at the same level, and so we find that with this smoothing level ( $\sim 1-3 \mathrm{kpc}$ around the Einstein radius), the particle noise does not affect the scattering of flux-ratio anomaly data points nor our statistics. In particular, here both $R_{\text {fold }}$ and $R_{\text {cusp }}$ are lower than 0.4, while in Figs 6 and 7 higher values are present in all the panels. We therefore conclude that the shot noise does not change our interpretation on the ray-tracing results presented in this paper and that the excess of flux-ratio anomalies seen for the simulated elliptical and disc galaxies is not due to particle noise, but to the presence of non-smooth structures in the lens galaxies, such as baryonic components.

\section{APPENDIX C: BARYONIC EFFECTS ON THE SHAPE OF CRITICAL CURVES}

The baryonic components in the lens galaxy can be the source of perturbations that contribute to the flux-ratio anomalies and also impact the shape of the critical curves. Fig. $\mathrm{C} 1$ demonstrates this effect by showing the young stars (black dots) overlapping with the critical curve (red curve) of a face-on disc lens, an edge-on disc lens, and an elliptical lens. We have seen in a few cases of face-on disc lenses that when the lens galaxy is massive enough, the critical curve can be distorted by the spiral arms. In the case of edge-on disc lenses, the critical curves are mostly elongated shaped due to the stellar disc with a high inclination angle. The elliptical lenses can show some level of distortions in the shape of their critical curves but in general retain their elliptical critical curves.
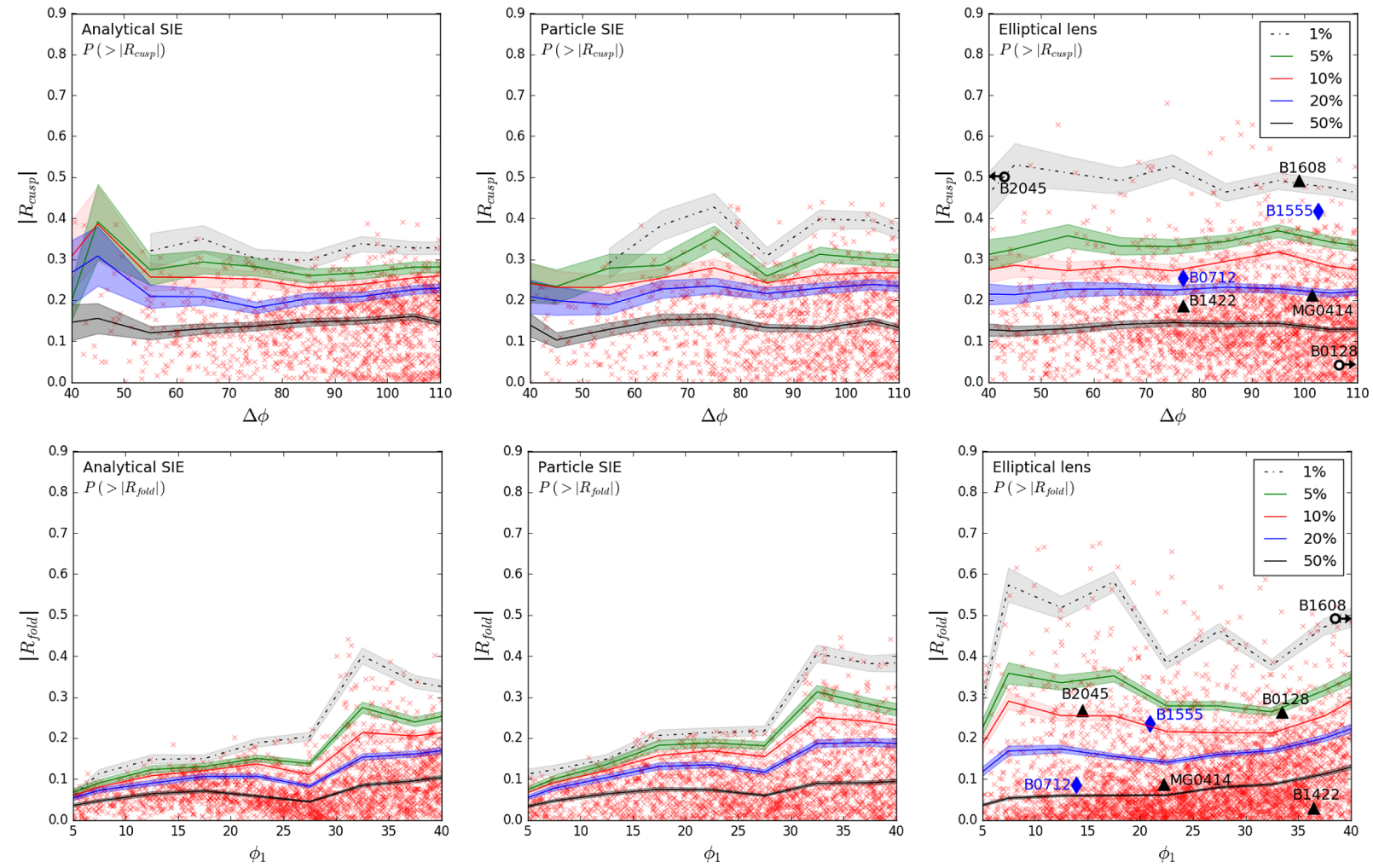

Figure B1. Flux-ratio anomaly strength distribution of analytical SIE, particle SIE halo (the smooth model results in Fig. 5), and the simulated elliptical lens ray-tracing results using GLAMER. The curves represent $1,5,10,20$, and 50 per cent of probability to find $\left|R_{\text {cusp }}\right|$ or $\left|R_{\text {fold }}\right|$ larger than a given value for a given opening angle $\left(\Delta \phi\right.$ or $\phi_{1}\left(^{\circ}\right)$ ), which the shaded area represents $1 \sigma$ uncertainty. The ray-traced data points are shown as red crosses. 

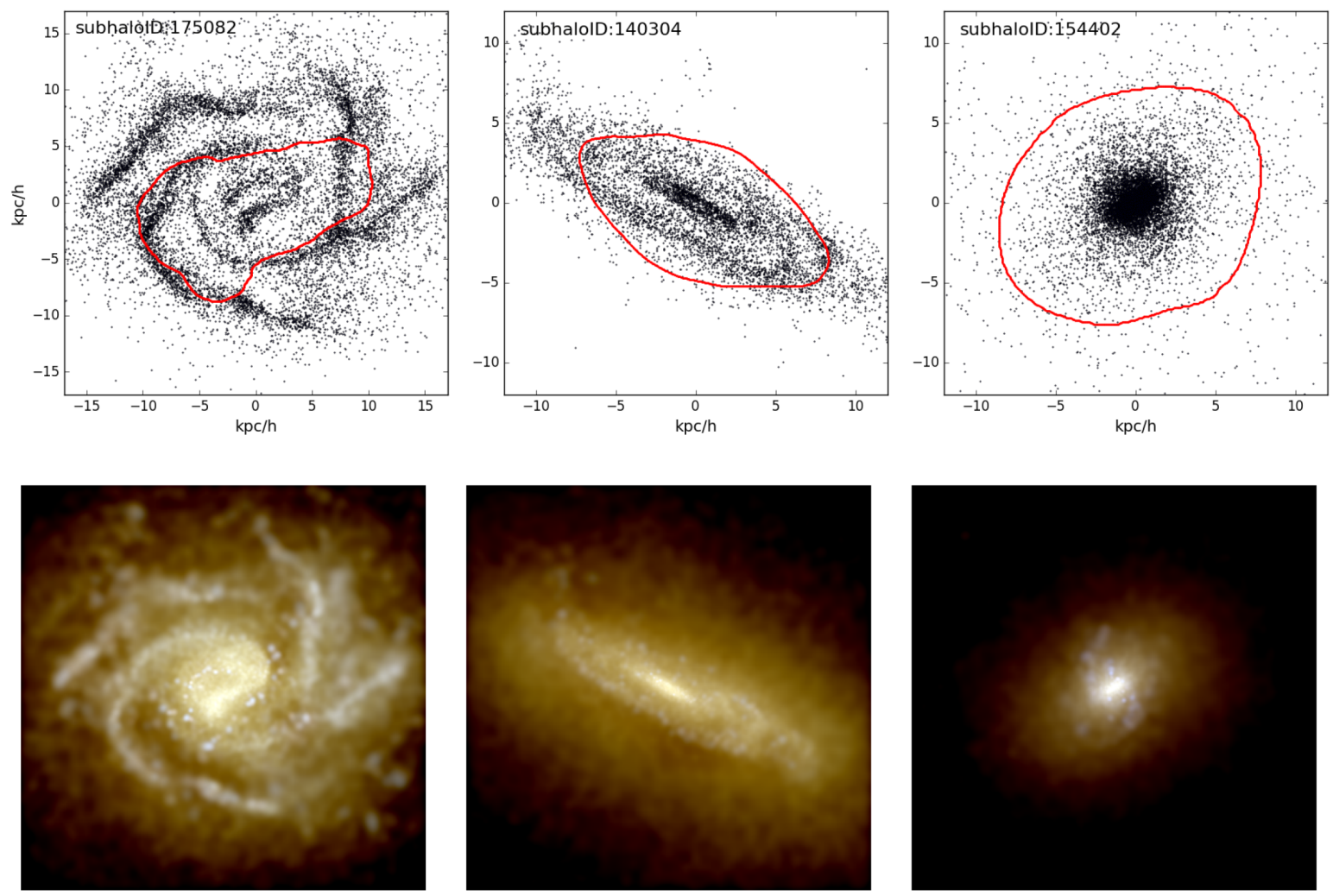

Figure C1. Upper: young stars (black dots) and critical curves (red curves) of a face-on disc lens, an edge-on disc lens, and an elliptical lens in our ray-tracing samples. The star particles plotted have less than $0.3 \mathrm{Gyr}$ of star formation time in disc galaxies and less than 2 Gyr in the elliptical galaxy. Note that the star particles in the Illustris simulation are not individual stars. The star formation time refers to the time when the star particle was formed once the local gas density excesses the certain threshold (see Vogelsberger et al. 2013, for more details). Bottom: synthesized images of each lens in the upper row with SDSS $g$, $r$, and $i$ filters (see Xu et al. 2017, for more details). The field of view of images is $15 \times 15 h^{-1} \mathrm{kpc}$.

This paper has been typeset from a $\mathrm{T}_{\mathrm{E}} \mathrm{X} / \mathrm{LAT} \mathrm{E}$ file prepared by the author. 